\title{
Development of classification models for basal stem rot (BSR) disease in oil palm using dielectric spectroscopy
}

\begin{abstract}
Basal stem rot (BSR) is the most destructive disease in oil palm plantations caused by Ganoderma boninense fungus, leading to a major economic setback in palm oil production. In order to reduce the losses caused by this disease, an effective early detection method is needed. Early detection not only prevents production losses, but it also reduces the use of chemicals. Therefore, this paper aims at investigating an early detection method utilizing dielectric properties (impedance, capacitance, dielectric constant, and dissipation factor) of oil palm trees. Leaf samples of healthy, mild, moderate, and severely-infected trees were collected and leaves' dielectric properties were measured at a frequency range of $100 \mathrm{kHz}-30 \mathrm{MHz}$ with $100 \mathrm{kHz}$ intervals. These spectral data were then reduced by principal component analysis (PCA) method. Following that, the reduced spectral data were tested to classify the leaf samples into four levels of disease severity. The classifiers used are linear discriminant analysis (LDA), quadratic discriminant analysis (QDA), k-nearest neighbor $(\mathrm{kNN})$, and Naïve Bayes (NB). The results showed that the dielectric spectra of oil palm leaves of diffident BSR severity levels were statistically different $(p<0.0004)$. In addition, despite the slight better performance of QDA classifier, ANOVA test revealed that there was no significant difference in accuracy between all other classifier models $(p=0.7169)$. Amongst the tested dielectric properties, impedance is considered the best parameter to assess the severity of BSR disease in oil palm with overall accuracy ranging from $81.82 \%$ to $100 \%$. These results verify the potential of dielectric spectroscopy for detecting BSR disease in oil palm.
\end{abstract}

Keyword: Basal stem rot; Dielectric properties; Oil palm; Quadratic discriminant analysis; Naïve Bayes; Dielectric spectroscopy 